\title{
Compression of Map Images by Multilayer Context Tree Modeling
}

\author{
Pavel Kopylov and Pasi Fränti
}

\begin{abstract}
We propose a method for compressing color map images by context tree modeling and arithmetic coding. We consider multicomponent map images with semantic layer separation and images that are divided into binary layers by color separation. The key issue in the compression method is the utilization of interlayer correlations, and to solve the optimal ordering of the layers. The interlayer dependencies are acquired by optimizing the context tree for every pair of image layers. The resulting cost matrix of the interlayer dependencies is considered as a directed spanning tree problem and solved by an algorithm based on the Edmond's algorithm for optimum branching and by the optimal selection and removal of the background color. The proposed method gives results $50 \%$ better than JBIG and $25 \%$ better than a single-layer context tree modeling.
\end{abstract}

Index Terms-Context modeling, image coding, layer ordering, spanning tree.

\section{INTRODUCTION}

D IGITAL maps are usually stored as vector graphics in a database for retrieving the data using spatial location as the search key. The visual outlook of maps representing the same region varies depending on the type of the map (topographic or road map), and on the desired scale (local or regional map). Vector representation is convenient for zooming as the maps can be displayed in any resolution defined by the user. The maps can be converted to raster images for data transmission, distribution via internet, or simply because of incompatibility of the vector representations of different systems. A compressed raster image format provides a reasonable solution in the form of compact storage size and compatible format.

Typical map images have high spatial resolution for representing fine details such as text and graphics objects but not so much color tones as photographic images. Thus, suitable compression method could be found among the lossless palette image compression methods such as GIF and PNG. The CompuServe graphics interchange format (GIF) is based on LZW dictionary compressor [1]. Portable network graphics (PNG) provides a patent-free replacement for GIF. It encodes the image using the deflate [2] algorithm, which is a combination of LZ77 dictionary compression [3] and Huffman coding.

On the other hand, lossy compression methods such as JPEG [4] are efficient for photographic images but do not apply well to palletized images and maps. The lossless JPEG-LS [5] uses

Manuscript received May 12, 2003; revised March 15, 2004. The associate editor coordinating the review of this manuscript and approving it for publication was Dr. Amir Said.

The authors are with the University of Joensuu, FI-80101 Joensuu, Finland (e-mail: justas@cs.joensuu.fi; franti@cs.joensuu.fi).

Digital Object Identifier 10.1109/TIP.2004.838694 linear predictive modeling, which also works well on natural images where adjacent pixels tend to have similar values. The predictive coding, however, is not efficient for images that contain only few colors.

It is also possible to divide the maps into separate color layers and to apply lossless binary image compression, such as JBIG and JBIG2 [6], [7]. They use context-based statistical modeling and arithmetic coding in the same manner as originally proposed in [8]. The probability of each pixel is estimated on the basis of context, which is defined as the combination of a set of already processed neighboring pixels. Each context is assigned with its own statistical model that is adaptively updated during the compression process. Decompression is a synchronous process with the compression.

Embedded image-domain adaptive compression of simple images (EIDAC) [9] uses the three-dimensional (3-D) context model tailored for the compression of grayscale images. The algorithm divides the image into bit planes and compresses them separately but context pixels are selected not only from the current bit plane, but also from the already processed layers.

Another approach which utilizes 3-D dependencies is called SKIP pixel coding [10]. Binary layers are acquired by color decomposition, and the coding sequence proceeds layer by layer. In a particular layer, if a given pixel has already been coded in a layer of higher priority, it does not need to be coded in the current layer or any of the lower layers. Thus, the coding of large amount of redundant information around blank areas could be "skipped."

Statistical context-based compression, such as the prediction by partial matching (PPM) [11], has also been applied to the compression of map images [12]. The method is a two-dimensional (2-D) version of the PPM method by combining a 2-D template with the standard PPM coding. The method has been applied to palette images and street maps. A simple scheme for resolution reduction has also been given and the proposed scheme was extended to resolution progressive coding, too.

The piecewise-constant image model (PWC) [13] is a technique designed for lossless compression of palette images, which uses a two-pass object-based modeling. In the first pass, the boundaries between constant color pieces are established. The color of the pieces are determined and coded in the second pass. The method was reported to give similar to or better compression than the JBIG but with a much faster implementation.

Among the alternative approaches, context-based statistical compression of the color layers is the most efficient in terms of compression. The location of the context pixels can be optimized for each layer separately as proposed in [14]. The method optimizes the location of the template pixels within a limited 


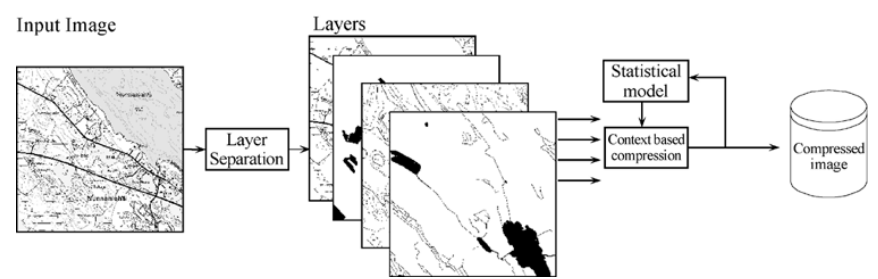

Fig. 1. System diagram to illustrate the compression method.

neighborhood area and produces the ordered template as the result. The ordering can then be used to derive the context template for any given template size. The method was then applied for generating optimized multilayer context template for map images [15].

Theoretically, better probability estimation can be obtained using a larger context template. The number of contexts, however, grows exponentially with the size of template; adding one more pixel to the template doubles the size of the model. This can lead to the context dilution problem where the statistics are distributed over too many contexts, thus affecting the accuracy of the probability estimates. The use of context tree [16] provides a more efficient approach for the context modeling so that a larger number of neighbor pixels can be taken into account without the context dilution problem.

In this paper, we propose a method for compressing multicomponent map images based on layer separation and context tree modeling. The main structure of the method is shown in Fig. 1. The paper is based on the ideas and results presented in two recent conference papers: the use of multilayer context tree model as proposed in [17] and the algorithm for obtaining optimal ordering of the layers as proposed in [18]. The acquiring of the optimal layer ordering is related to the directed spanning tree problem, and is solved by an algorithm derived from the Edmond's algorithm for optimum branching.

The rest of the paper is organized as follows. Context-based compression and context tree modeling are recalled in Section II. Compression of map images is then considered in Section III for semantic and color separated layers. The multilayer template, fixed-size template, and multilayer context tree models are studied. The problem of optimal ordering of the layers is studied in Section IV. Experiments are given in Section V, and conclusions are drawn in Section VI.

\section{CONTEXT-BASED COMPRESSION}

Statistical image compression consists of two distinct phases: statistical modeling and coding [19]. In the modeling phase, we estimate the probability distribution of the symbols to be compressed. The coding process assigns variable length code words to the symbols according to the probability model so that shorter codes are assigned to more probable symbols, and vice versa. The coding can be performed using arithmetic coding, which provides optimal coding for the given probability model [20].

\section{A. Statistical Modeling}

A binary image can be considered as a message generated by an information source. The idea of statistical modeling is to

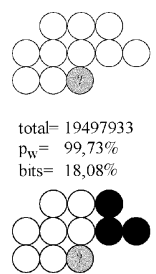

total $=121745$ $\mathrm{p}_{\mathrm{w}}=86,99 \%$ bits $=2,32 \%$

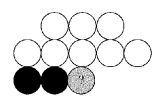
$\mathrm{p}_{\mathrm{w}}=11,68 \%$ bits $=6,83 \%$

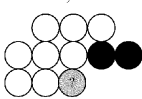

total $=69926$ $\mathrm{p}_{\mathrm{w}}=61,99 \%$
bits $=2,29 \%$ total $=383952$
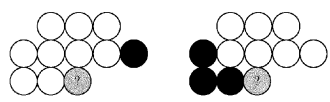

total $=297123$ $\mathrm{p}_{\mathrm{w}}=93,40 \%$
bits $=3,56 \%$ bits $=3,56 \%$
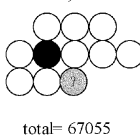

$\mathrm{p}_{\mathrm{w}}=52,79 \%$ bits $=2,29 \%$

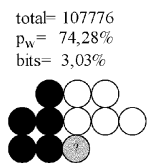

total $=82643$ $\mathrm{p}_{\mathrm{w}}=77,58 \%$ bits $=2,17 \%$

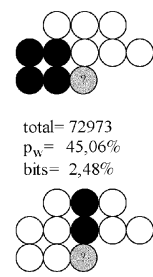

total $=65772$ $\mathrm{p}_{\mathrm{w}}=37,63 \%$ bits $=2,15 \%$
Fig. 2. Most important contexts with the ten-pixel template of JBIG. The results are for the basic layer of the image 431306 with semantic separation (see Section V).

describe the message symbols (pixels) according to the probability distribution of the source alphabet (binary alphabet, in our case). Shannon has shown in [21] that the information content of a single symbol (pixel) in the message (image) can be measured by its entropy.

The pixels in an image form geometrical structures with appropriate spatial dependencies that can be described by context-based statistical model [8]. The probability of a pixel is conditioned on a context $C$, which is defined as the black-white configuration of the neighboring pixels within a local template. The entropy of an $N$-level context model is the weighted sum of entropies of individual contexts

$$
H_{N}=-\sum_{j=1}^{N} p\left(C_{j}\right) \cdot\left(p_{w}^{C_{j}} \cdot \log _{2} p_{w}^{C_{j}}+p_{b}^{C_{j}} \cdot \log _{2} p_{b}^{C_{j}}\right)
$$

where $p\left(C_{j}\right)$ is the probability of the context $C_{j}$ and $p_{w}^{C_{j}}$ and $p_{b}^{C_{j}}$ are the probabilities of the white and black pixel in the context $C_{j}$, respectively.

Nevertheless, only a small fraction of all contexts are really important. For example, in the case of sample binary images with ten-pixel context template, about 50\% of the code bits originate from the few most important contexts as illustrated in Fig. 2. Furthermore, $99 \%$ of the code bits originate from 183 contexts, and 429 out of the 1024 contexts are never used at all.

\section{B. Context Tree}

Context tree provides a more flexible approach for modeling the contexts so that larger number of neighbor pixels can be taken into account without the context dilution problem [16]. The contexts are represented by a binary tree, in which the context is constructed pixel by pixel. The context selection is deterministic and only the leaves of the tree are used. The location of the next neighbor pixels and the depth of the individual branches of the tree depend on the combination of the already coded neighbor pixel values. Once the tree has been created, it is fully static and can be used in the compression as any other fixed-size template.

Context tree is applied in the compression in a similar manner as the fixed-size context templates; only the context selection is different. The context selection is made by traversing the context tree from the root to leaf, each time selecting the branch according to the corresponding neighbor pixel value. The leaf has a pointer (index) to the statistical model that is to be used. 
Each node in the tree represents a single context. The two children of a context correspond to the parent context augmented by one more pixel. The position of this pixel can be fixed in a predefined order, or optimized within a limited search area, relative to the compressed pixel position.

The tree can be optimized beforehand using a training image (static approach) [22] or optimized directly to the image to be compressed (semi-adaptive approach) [16]. In the latter case, an additional pass over the image is required to collect the statistics, and the tree must also be stored in the compressed file. The cost of storing the tree structure is one bit per node. The static approach is possible because of the similarity of the trees with images of the same type. On the negative side, the resulting tree would be more dependent on the choice of the training image.

\section{Construction of the Tree}

To construct a context tree, the image must be processed and statistics should be calculated for potential contexts in the tree including the internal nodes. The tree must then be pruned by comparing the parent node and its two sub trees at every level. If compression gain is not achieved by using the two sub trees instead of the parent node, the sub trees should be removed and the parent node would be a leaf node. The compression gain is calculated as

$$
\operatorname{Gain}(C)=l(C)-l\left(C_{\text {left }}\right)-l\left(C_{\text {right }}\right)-\text { SplitCost }
$$

where $C$ is the parent node, and $C_{\text {left }}$ and $C_{\text {right }}$ are the two sub trees. The code length $l$ denotes the total number of output bits from the pixels coded using the context in the particular node. The cost of storing the tree is integrated into the SplitCost. The code length can be calculated by summing up the self-entropies of the pixels as they occur in the image

$$
l(C)=-\sum_{t} \log p^{t}(C)
$$

where $p^{t}(C)$ is the probability of upcoming symbol within context $C$ at time moment $t$. The probability of the pixel is calculated on the basis of the observed frequencies using a Bayesian sequential estimator

$$
p^{t}(C)= \begin{cases}p_{w}^{t}(C)=\frac{n_{w}^{t}(C)+\delta}{n_{w}^{t}(C)+n_{w}^{t}(C)+2 \delta}, & \text { if } t \text { th pixel is white } \\ p_{b}^{t}(C)=1-p_{w}^{t}(C), & \text { if } t \text { th pixel is black }\end{cases}
$$

where $n_{w}^{t}$ and $n_{b}^{t}$ are time-dependent frequencies and $p_{w}^{t}$ and $p_{b}^{t}$ are the probabilities for white and black colors, respectively, and $\delta=0.45$, as in [6].

The tree could be constructed in a straightforward manner by applying the pruning criterion locally but the tree could then terminate too early as in the example shown in Fig. 3. It is, therefore, recommended that the tree is optimized globally as follows.

The construction of the context tree consists of two parts [23]: choosing an order of the context pixels and constructing optimal context tree for the given order. The following construction strategies have been considered:

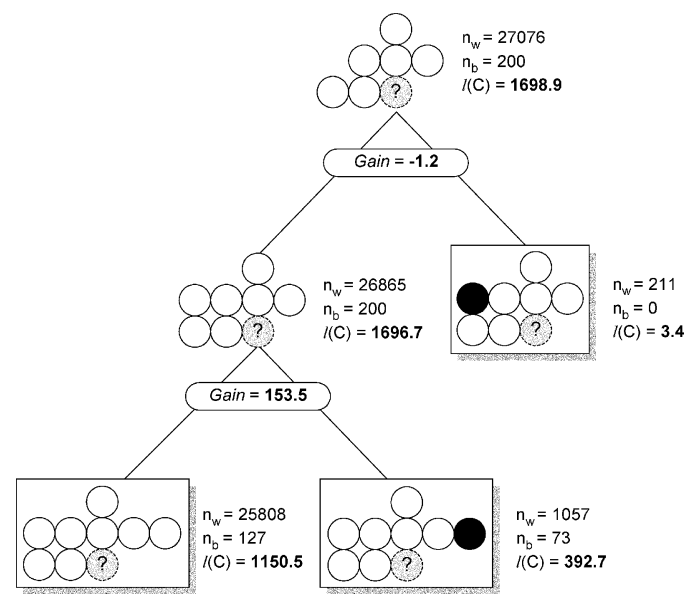

Fig. 3. Example of tree pruning with local pruning criterion. The code length is calculated adaptively according to (2).

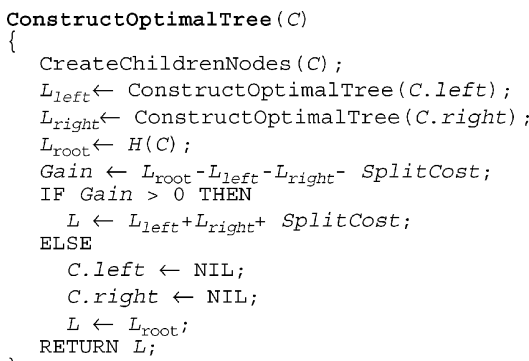

Fig. 4. Recursive algorithm for optimal construction of the context tree.

1) Optimal bottom-up: The approach constructs a full tree of $k$ levels, which is then pruned one level at a time using the criterion of (1). The bottom-up approach provides optimal context tree [24] for the given order but at the cost $O\left(2^{k}\right)$ time and space.

2) Optimal top-down: The tree is constructed by recursively expanding the tree starting from the root using the algorithm in Fig. 4. The advantage is that only linear time and space complexity is needed [23].

3) Free-tree: The tree is constructed stepwise by expanding the tree one level at a time using a local pruning criterion. The location of the context pixel is optimized for each node separately [16].

In a static approach, there is no overhead from storing the tree and the SplitCost is 0 . In the semi-adaptive approach, the cost for storing the tree is $1 \mathrm{bit} /$ node when fixed context order is used. In the case of free tree, the position of the next context pixel must also be stored for each context. The additional cost of storing the location of this split pixel is also integrated into SplitCost parameter.

\section{MAP IMAGE COMPRESSION}

Map images can be a result of rasterization of vector map format such as simple vector format (SVF), scalable vector graphics (SVG), or ERSI ArcShape [25]. The map server can provide the maps as a set of layers with different semantic meaning. For example, the topographic map series 1:20 000 


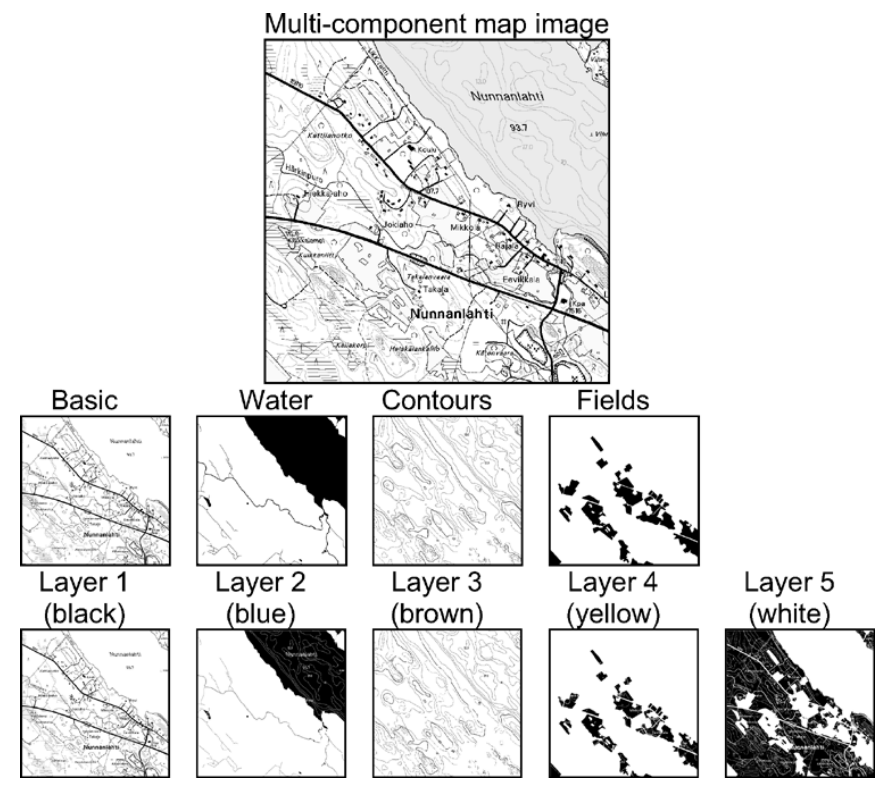

Fig. 5. Illustration of a multicomponent map image. The shown fragment has the dimensions of $1000 \times 1000$ pixels.

of National Land Survey of Finland (NLS) [26] divides the information into four logical layers: basic (topographic data and contours), elevation lines, fields, and water. The size of each layer is $5000 \times 5000$ pixels, and represents a $10 \times 10 \mathrm{~km}^{2}$ area. The map image can then be easily reconstructed by combining the binary layers, and displayed to the user as a color image.

\section{A. Representation of Map Image}

In order to utilize the context-based compression, the map must be divided into binary layers. Each layer is compressed separately, and the compressed layers are stored into the same file, as proposed in [27]. There are three alternative approaches to do this: semantic separation, color separation and bit-plane separation.

This first approach, semantic separation, means that the map is output into a set of binary layers each containing different semantic meaning. This representation is possible if the maps are obtained directly from a map database. The advantages of semantic separation are better compressibility, and that the layers to be shown can be selected at the time of viewing.

On the other hand, the map image could be provided as a raster color image without any additional information about the semantics of the image. In this case, color separation must be used to divide the image into binary layers so that each layer represents one color of the original image. The drawback of the color separation is that information of the original semantic separation cannot be recovered. Furthermore, the color separation can fragment the binary layers, as shown in Fig. 5. For example, overlapping text elements break the continuation of fields and lakes. This does not decrease the quality of the image but it increases the complexity of these layers, and, thus, the compressed file size. Moreover, the color separation results into one additional layer-the background color.

The third approach, bit-plane separation, must be applied when we have the original map only as a raster image, and the number of colors is too high for efficient color separation. For

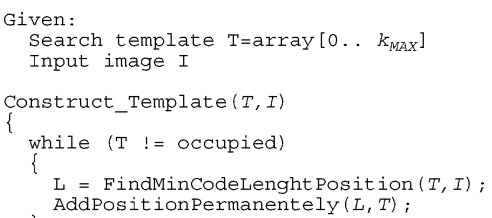

Fig. 6. Algorithm for optimizing the context template.
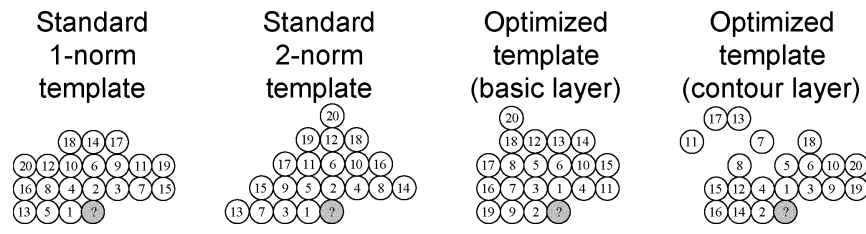

Fig. 7. Alternative orderings for the context templates.

example, the image might have been digitized from a paper copy and stored using lossy compression method, such as JPEG [4]. In the bit-plane separation, the number of colors is first reduced by quantizing the image into a limited size color palette, or to 256 gray scales. The resulting image is then separated into bit planes using Gray coding [28] and represented as a sequence of binary images. In this paper, we consider only the first two approaches (semantic and color separation).

\section{B. Independent Compression of Layers}

Straightforward solution is to compress the layers separately by fixed-size template defined by the standard 1-norm and 2-norm distance functions (see Fig. 7). The optimal template size depends on the size of the image. The location of the template pixels, on the other hand, has no direct effect on the learning cost but, if properly designed, may greatly improve the accuracy of the model. It is, therefore, feasible to optimize the location of the template pixels for the compressed images.

It is possible to optimize the size and shape of the template for the given image layer to be compressed at the cost of longer compression time [14]. The optimal context template can be solved by compressing the image using all possible templates and selecting the one with the most compression. However, there are an exponential number of different template configurations as a function of the template size. A more practical approach is to optimize the location of the template pixels one pixel at a time as outlined in Fig. 6. Alternative orderings for the context templates are outlined in Fig. 7.

The optimization starts with an empty context template and expands it by one pixel at a time. A new pixel is added to each unoccupied location in the neighborhood area. For each candidate pixel location, we calculate the statistics of the image and compare the entropies of the model with different location of the candidate pixel. We then select the pixel location providing minimum entropy and add it permanently to the context template. The process is continued until the context template size reaches a predefined maximum context size $k_{\text {max }}$.

The optimization can be applied in two alternative manners: static and semi-adaptive. In the static approach, we optimize the template using a priori knowledge of the image type. This is possible, as we know the type of the images to be compressed. The 


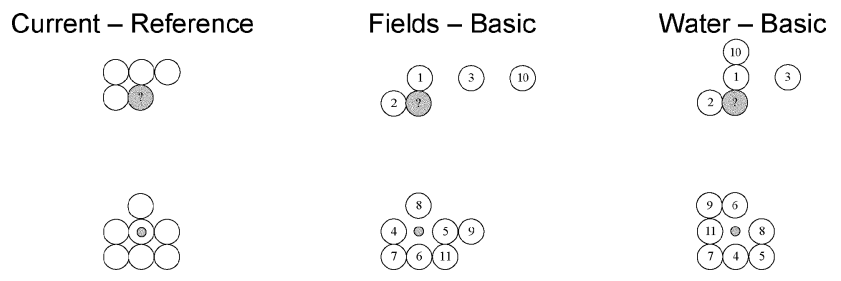

Fig. 8. (Left) Sample two-layer context templates for JBIG-2, (middle) optimized for fields layer, and (right) for water layer when basic is used as the reference layer.

advantage is that the time-consuming optimization can be done off line. In the semi-adaptive approach, the template is optimized for the compressed image and is stored in the compressed file. This is a better solution if the image type is not known beforehand. The compression process, however, would be very slow, which makes this approach not suitable for real-time applications.

\section{Optimized Multilayer Template}

The idea of multilayer context template is to utilize the information from additional image layer, referred here as the reference image. The restriction on the use of the reference image is that it must have already been coded so that both encoder and decoder have the same information. The main difference in the construction of single-layer and multilayer context templates is in additional neighborhood mask used for selection of the pixels from the reference image. The pixels in the current layer must be already coded pixels, but in the reference layer, the pixels can be anywhere in the image.

The idea of utilizing interlayer dependencies have been used in JBIG2 [7]. Two-layer context template is applied for utilizing the information of a matching symbol when compressing the pixels in the segment. The same idea was then applied for generating multilayer context template for map images [15]. In [29], gray-scale images are divided into binary layers by Gray coding and bit-plane separation. The bit planes are then compressed as binary images using context template including pixels both from the current layer and from the pixels in the previously coded (higher layer) bit planes.

In the following, we use the combined neighborhood area of 77 pixels, which was introduced in [17]. Optimized sample context templates for the map images are shown in Fig. 8. The two-layer context template of JBIG2 is shown for comparison.

\section{Multilayer Context Tree}

The idea of utilizing multilayer dependencies can be extended also to the context tree modeling. The multilayer context tree is constructed as follows. The tree starts from scratch and the branches are expanded one pixel at a time. The location of the template pixels are optimized and fixed beforehand and then applied for every branch. Another approach is to optimize the location separately for every branch (free tree approach). The context pixels are chosen from the same joint 77 pixels neighborhood.

The use of the information from the reference layer will allow us in some cases to increase the compression ratio of the single layer up to 50\% according to [17]. In fact, if we will consider

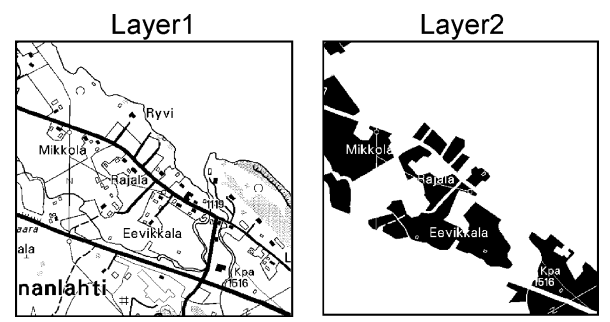

Fig. 9. Example of two layers obtained by color separation.

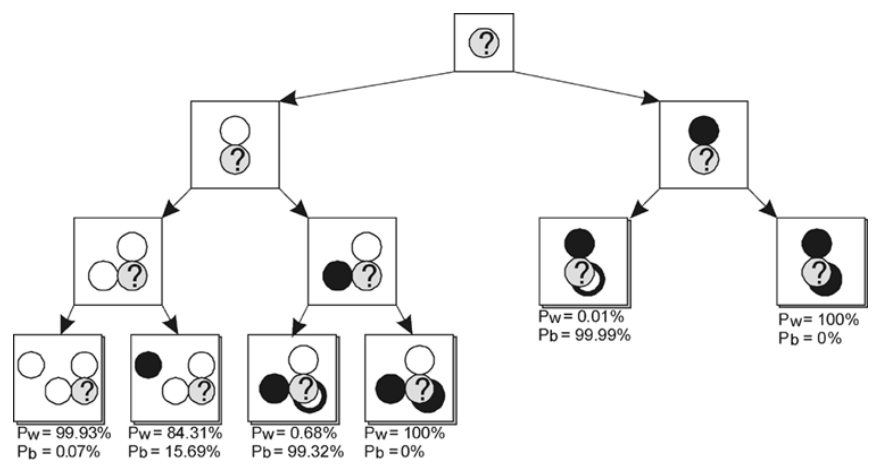

Fig. 10. Example of a two-layer context tree, in which two context pixels are taken from the current layer and one from the reference layer (shown below the current pixel).

the compression of sample images in Fig. 9, the compression of these two images separately using single-layer context trees would result in $4854+1330=6184$ bytes. On the other hand, if we use the information from the first layer when compressing the second layer, the tree structure of the second layer would be simpler. All information would be concentrated only in the first branch of the tree, as shown in Fig. 10. Thus the compression of the second layer would be only 146 bytes, and the final size of the compressed file $4854+146=5000$ bytes.

The map images usually have interlayer dependencies. For example, the same pixel is usually not set in the water layer and in the field layers at the same time, although it is possible as the layers are generated from the map database independently from each other. Another observation is that the basic and the water layers have redundant information along the rivers and lake boundaries. In general, anything is possible, and it is not easy to observe the existing dependencies by the eye. The dependencies, however, can be automatically captured by the statistical modeling.

\section{OPTIMAL ORDERING OF THE LAYERS}

The existing dependencies are demonstrated in Fig. 11, in the case of the NLS map images. There are significant interlayer dependencies between the basic layer and the two other layers (water and field). The contour layer, on the other hand, is independent from all other layers. The main observation is that we cannot utilize all the existing dependencies as the order of processing restricts which layers we can use as the reference layer.

For example, if we compress the basic layer first, we can then improve the compression of the water layer by $52 \%$ (118705 bytes). The opposite order would improve the compression of the basic layer by $35 \%$ (345061 bytes). It is easy to see that the 


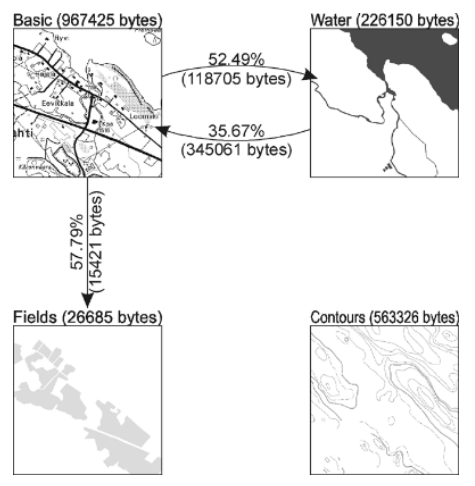

Fig. 11. Arrows show the interlayer dependencies as the number of saved bits when compressing the second image using the first one as reference image.

TABLE I

EXAMPLE OF THE COST MATRIX FOR A SAMPLE IMAGE

\begin{tabular}{c|c|c|c|c|c|c|c|c|c|c|c|c}
\multicolumn{2}{c|}{0} & 1 & 2 & 3 & 4 & 5 & 6 & 7 & 8 & 9 & 10 & 11 \\
\hline 0 & & 7564 & 13013 & 6189 & 1792 & 10448 & 16472 & 28477 & 1619 & 13683 & 13360 & 23976 \\
\hline 1 & 17158 & & 16041 & 5871 & 1731 & 9997 & 15713 & 27988 & 1557 & 11994 & 11377 & 22000 \\
\hline 2 & 13488 & 8594 & & 6029 & 1834 & 10461 & 16488 & 27318 & 1946 & 13671 & 13105 & 23760 \\
\hline 3 & 23390 & 12116 & 20700 & & 1636 & 9931 & 12333 & 28093 & 2055 & 13567 & 12735 & 22189 \\
\hline 4 & 23471 & 12789 & 21348 & 6091 & & 11011 & 18168 & 30445 & 2239 & 13683 & 12682 & 23713 \\
\hline 5 & 23293 & 12116 & 21144 & 5385 & 1751 & & 15506 & 26732 & 1834 & 13214 & 12102 & 22548 \\
\hline 6 & 23536 & 12269 & 21423 & 3934 & 1681 & 10134 & & 27405 & 2111 & 13674 & 13385 & 20479 \\
\hline 7 & 20197 & 10149 & 17117 & 4889 & 1646 & 7007 & 12446 & & 1737 & 11075 & 10612 & 13574 \\
\hline 8 & 22810 & 11956 & 22493 & 6780 & 1834 & 10617 & 16899 & 29489 & & 13256 & 13097 & 23976 \\
\hline 9 & 26015 & 12029 & 20013 & 6467 & 1834 & 10329 & 16494 & 29514 & 2239 & & 9401 & 22764 \\
\hline 10 & 23036 & 11617 & 19305 & 5628 & 1722 & 10154 & 15797 & 27815 & 2013 & 7344 & & 22038 \\
\hline 11 & 26169 & 12561 & 21008 & 5933 & 1829 & 10660 & 14706 & 23261 & 1963 & 12231 & 12515 & \\
\hline
\end{tabular}

best order of these layers would be to compress first the water layer, second the basic layer, and then the fields layer last. The contours layer should be processed independently.

In general, we can select any predefined order on the basis of known (or assumed) dependencies. If the image source is not known beforehand, we should find the optimal order of the layers for maximal utilization of the interlayer dependencies. The selected processing order can be saved in the compressed file using a few bits. The problem of finding the optimal order is studied in the following subsections.

\section{A. Construction of the Cost Matrix}

Suppose that we have $k$ layers. In order to determine the optimal layer ordering, we have to consider all pairwise dependencies by tentatively compressing every layer using all other layers as reference. This results in a $k \times k$ cost matrix consisting of the absolute bit rates for every layer-reference layer pairing (see Table I). On the basis of the cost matrix, we can generate all $k$ ! possible permutations for the processing order and calculate the total saving achieved by the given layer ordering. If the number of layers is small enough (with the NLS images $k=4$ ), this is not a problem. With larger values of $k$, however, this could be computationally too expensive.

On the other hand, not all information in the matrix is relevant to us. In the case when there are no dependencies between the layers, the corresponding compression result would be the same (or worse) with or without the use of interlayer context model. We can, therefore, reduce the amount of information in the cost matrix by subtracting the original values (Table II) by the values obtained by layer-independent compression (Table I), and eliminate values smaller than or equal to zero. The resulting cost matrix is shown in Table III.
TABLE II

ORIGINAL COMPRESSION VALUES OBTAINED WITHOUT THE USE OF DEPENDENCIES

\begin{tabular}{c|c|c|c|c|c|c|c|c|c|c|c}
\hline 0 & 1 & 2 & 3 & 4 & 5 & 6 & 7 & 8 & 9 & 10 & 11 \\
\hline 23451 & 11847 & 20123 & 5504 & 1734 & 10256 & 15341 & 29206 & 1845 & 12328 & 11495 & 23049 \\
\hline
\end{tabular}

TABLE III

EXAMPLE OF THE COST MATRIX AFTER REDUCTION

\begin{tabular}{c|c|c|c|c|c|c|c|c|c|c|c|c}
\multicolumn{2}{c|}{0} & 1 & 2 & 3 & 4 & 5 & 6 & 7 & 8 & 9 & 10 & 11 \\
\hline 0 & & 4283 & 7110 & 0 & 0 & 0 & 0 & 729 & 226 & 0 & 0 & 0 \\
\hline 1 & 6293 & & 4082 & 0 & 3 & 259 & 0 & 1218 & 288 & 334 & 118 & 1049 \\
\hline 2 & 9963 & 3253 & & 0 & 0 & 0 & 0 & 1888 & 0 & 0 & 0 & 0 \\
\hline 3 & 61 & 0 & 0 & & 98 & 325 & 3008 & 1113 & 0 & 0 & 0 & 860 \\
\hline 4 & 0 & 0 & 0 & 0 & & 0 & 0 & 0 & 0 & 0 & 0 & 0 \\
\hline 5 & 158 & 0 & 0 & 119 & 0 & & 0 & 2474 & 11 & 0 & 0 & 501 \\
\hline 6 & 0 & 0 & 0 & 1570 & 53 & 122 & & 1801 & 0 & 0 & 0 & 2570 \\
\hline 7 & 3254 & 1698 & 3006 & 615 & 88 & 3249 & 2895 & & 108 & 1253 & 883 & 9475 \\
\hline 8 & 641 & 0 & 0 & 0 & 0 & 0 & 0 & 0 & & 0 & 0 & 0 \\
\hline 9 & 0 & 0 & 110 & 0 & 0 & 0 & 0 & 0 & 0 & & 2094 & 285 \\
\hline 10 & 415 & 230 & 818 & 0 & 12 & 102 & 0 & 1391 & 0 & 4984 & & 1011 \\
\hline 11 & 0 & 0 & 0 & 0 & 0 & 0 & 635 & 5945 & 0 & 97 & 0 & \\
\hline
\end{tabular}

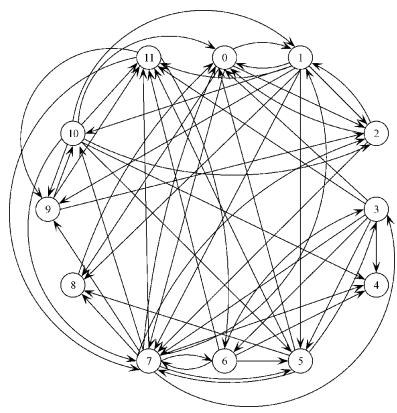

Fig. 12. Graph representation of Table III.

The reduced cost matrix can be considered as a directed graph with $k$ nodes as shown in Fig. 12. The problem of finding the optimal order is closely related (but not exactly) to the minimum spanning tree problem. We will next give graph-based algorithm to solve the optimal ordering. We follow the approach taken by Tate for optimal band ordering in the compression of multispectral images [30].

\section{B. Maximum Spanning Tree}

A spanning tree of a graph is a subset of the edges so that all nodes of the graph are included in the set but there are no cycles. Minimum spanning tree (MST) is a spanning tree with the minimum sum of the weights of the edges included in the given graph [31].

The minimum weighted tree can be solved in polynomial time using Prim's algorithm [32], for example. The algorithm begins by adding the lowest weighted edge into the solution. It then loops by adding the next lowest weighted edge that connects a node inside the set to another node outside of the set. The algorithm terminates when $n-1$ edges have been chosen and, thus, all nodes covered.

However, there are few differences that separate our problem of obtaining the optimal order from the minimum spanning tree problem.

1) We have a directed graph whereas the MST is defined with undirected graph.

2) We can have only one incoming edge for any node.

3) We can have several separate spanning trees instead of only one. 


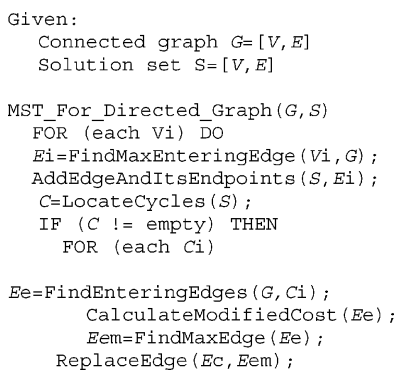

Fig. 13. Edmond's algorithm.

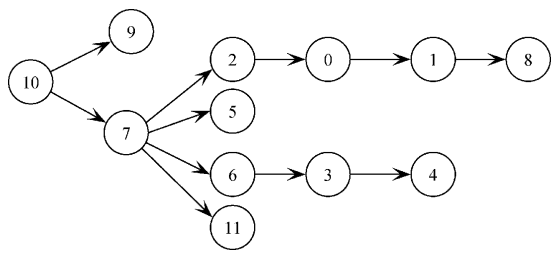

Fig. 14. Resulting spanning tree after Edmonds' algorithm.

4) We have maximization problem.

The first two differences make the problem as a directed spanning tree problem. The directed spanning tree is defined as a spanning tree where all nodes (except the root) have exactly one incoming edge. This is also known as the optimum branching problem [33] and can be solved in $O\left(n^{2}\right)$ time [34].

In the optimal ordering, it is not necessary to have a single spanning tree, but we can have separate sub graphs, see Fig. 11. This means that we should actually find spanning forest instead of a single tree. The problem was considered as the maximum spanning forest problem in [30]. However, we have eliminated all negative weights in the cost matrix (Table III), and the inclusion of a zero-edge can be considered as independent compression of the corresponding layers. Thus, we can still consider the optimal ordering as directed spanning tree problem.

We apply Edmond's algorithm [33], as shown in Fig. 13. The algorithm begins by selecting the maximum incoming edge for every node except the root. If no cycles are formed, the resulting graph is MST. Otherwise, the algorithm detects and removes existing cycles by removing an edge in the cycle and replacing it by another edge incoming from outside. The edges are chosen so that maximal saving is achieved. We also note that the Edmond's algorithm requires that one node is selected as the root. We, therefore, repeat the algorithm using every node as potential root and select the one resulting to most saving. The time complexity of the algorithm for a single root is $O\left(n^{2}\right)$ and for considering all nodes as a root $O\left(n^{3}\right)$.

The optimal branching for the data in Table I is shown in Fig. 14. This ordering of the layers sums up to 124977 bytes, which corresponds to the improvement of $24.79 \%$ in comparison to the original result. The process of the algorithm in detail is illustrated in Fig. 15 using node 10 as the root. The initial solution contains three cycles $(0 \leftrightarrow 2,3 \leftrightarrow 6$, and $7 \leftrightarrow 11)$. The first cycle $(0 \leftrightarrow 2)$ is eliminated by replacing the edge $0 \rightarrow 2$ by the edge $1 \rightarrow 2$. This creates a new cycle $(0 \rightarrow 1 \rightarrow 2)$, which is then resolved in the second step by replacing the edge $1 \rightarrow 2$ by the edge $7 \rightarrow 2$. The rest of the cycles are resolved accordingly.

\section{Selection of the Background Color}

In the case of color separation, we can eliminate one layer completely and consider it as the background color. The background color is usually white but this is not necessarily the case always. In fact, we can set any layer as the background color. The advantage is that the chosen layer is not stored in the compressed file at all. There are two obvious choices for selecting the background color:

1) greedy: the layer with the maximal compressed size;

2) optimal: the layer of whose removal gives most improvement in compression.

The greedy choice is not necessary the best choice because the layer with most information can also include most interlayer dependencies with other layers. In other words, the background layer cannot be used as a reference layer, and, therefore, the removal of the dependent layer can increase the compressed size of other layers.

The optimal choice can be obtained by considering all layers as the background color and selecting the one that result in the best overall compression. In principle, this is computationally demanding as the problem of finding the optimal ordering takes $O\left(n^{3}\right)$, and, thus, $O\left(n^{4}\right)$ time for finding the optimal choice for the background color. In practice, however, the number of layers is small (here, $n=4 \ldots 16$ ). Therefore, the bottleneck of the optimization is not the solving of the spanning tree but the calculation of the cost matrix at the first place.

\section{EXPERIMENTS}

We evaluate the proposed method by compressing the sets of map images listed in Table IV, and illustrated in Fig. 16. The sets \#1 to \#4 are from the map database of the National Land Survey of Finland (NLS) [26]. In the case of the detailed road maps (Sea and City), the number of colors was reduced to 16 before the compression.

Two different image representations are considered: 1) semantic representation and 2) color separation. Semantic representation was available for five randomly selected map sheets of scale $1: 20000$. These images are further denoted as 124101, 201401, 263112, 431204, and 431306 according to their map sheet number in the NLS database. The rest of the images were passed trough color separation. The obtained binary layers (semantic or color separation) were compressed using semi-adaptive context tree modeling and arithmetic coding. The optimal ordering and the choice of background color were solved for every image separately. The context trees were created using the algorithm in Fig. 3.

We first study the effect of the multilayer context tree modeling by compressing the set of images by the following variants:

1) CT: context tree modeling of each layer separately;

2) MCT (single): multilayer context tree using single conditioning layer;

3) MCT (optimized): multilayer context tree using optimal layer ordering.

All variants are considered with and without optimal selection of the background color. In the semantic separation, the 


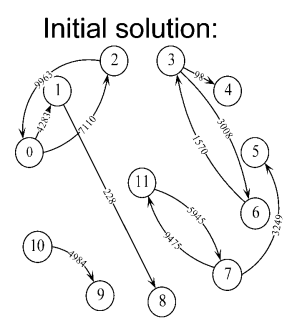

Second cycle:

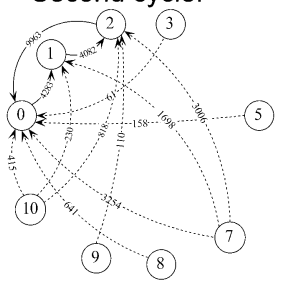

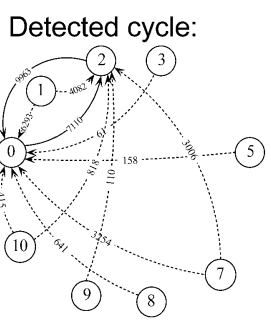

Cycle removed:

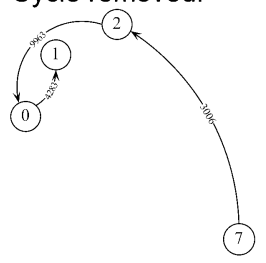

Cycle removed:

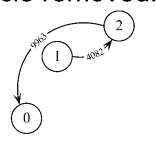

Final solution:

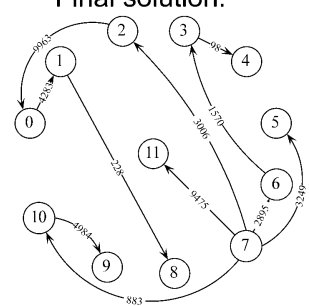

Fig. 15. Process of Edmond's algorithm. The first graph is the result of selecting maximum incoming edge except the root (initially node 10). The following steps illustrate the removal of cycles and the final result.

TABLE IV

SET OF TEST IMAges

\begin{tabular}{|c|c|c|c|c|c|}
\hline & Scale & Type & Images & Image size & No. of colors \\
\hline$\frac{\text { Set \#1: }}{\text { semantic separation }}$ & $1: 20000$ & Topographic & 5 & $5000 \times 5000$ & 5 \\
\hline Set \#1: & 1.20000 & Topogranhic & 5 & $5000 \times 5000$ & 6 \\
\hline color separation & & & & $5000 \times 5000$ & \\
\hline Set \#2: & $1: 8000$ & Topographic & 4 & $1024 \times 1024$ & 7 \\
\hline Set \#3: & $1: 100000$ & Road map & 4 & $1024 \times 1024$ & 16 \\
\hline Set \#4: & $1: 800000$ & Road map & 4 & $1024 \times 1024$ & 16 \\
\hline Set \#5: & $1: 250000$ & $\begin{array}{l}\text { Road map } \\
\text { (detailed) }\end{array}$ & 2 & $800 \times 800$ & 16 \\
\hline
\end{tabular}

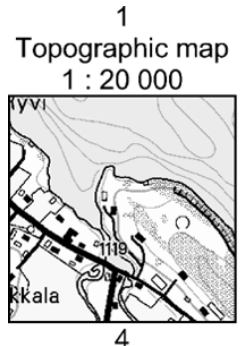

Road map

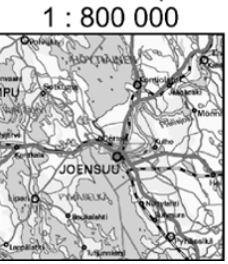

2

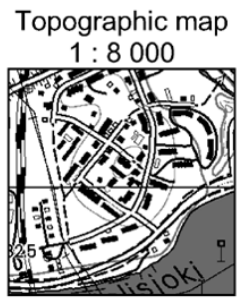

Detailed - Sea $1: 250000$

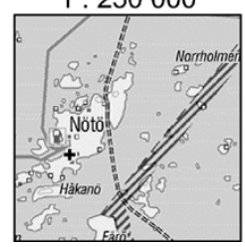

3

Road map $1: 100000$
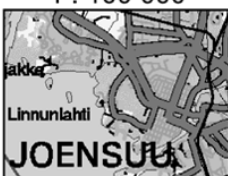

5
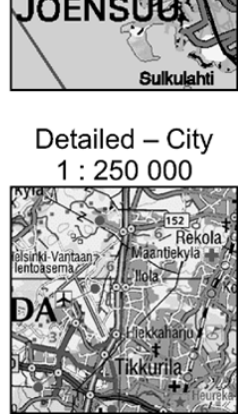

Fig. 16. Sample $256 \times 256$ pixel fragments of the test images.

background is excluded by default, as it was never included. The results show that the multilayer context tree (MCT) produces file sizes of about $25 \%$ less than the single layer context tree (CT) (see Table V). The optimal ordering is about $10 \%$ better than the use of a single conditioning layer.

The compression performance is illustrated as a function of the number of contexts in Fig. 17, and compared to the fixed size context modeling (JBIG), single-layer context tree (CT),
TABLE $\mathrm{V}$

AVERAGE COMPRESSION RESULTS (KILOBYTES) FOR THE SET OF TEST IMAGES

\begin{tabular}{|c|c|c|c|c|c|c|}
\hline & \multicolumn{3}{|c|}{ With background } & \multicolumn{3}{|c|}{ Without background } \\
\hline & CT & MCT single & $\begin{array}{c}\text { MCT } \\
\text { optimized }\end{array}$ & CT & MCT single & $\begin{array}{c}\text { MCT } \\
\text { optimizec }\end{array}$ \\
\hline $\begin{array}{c}\text { Set \#1 } \\
\text { semantic separation }\end{array}$ & $\cdots$ & -- & -- & 441 & 395 & 382 \\
\hline $\begin{array}{c}\text { Set \#1 } \\
\text { color separation }\end{array}$ & 960 & 612 & 577 & 616 & 495 & 401 \\
\hline Set \#2 & 64 & 37 & 36 & 34 & 32 & 30 \\
\hline Set \#3 & 243 & 211 & 194 & 185 & 173 & 155 \\
\hline Set \#4 & 268 & 240 & 219 & 240 & 217 & 198 \\
\hline Set \#5 & 198 & 165 & 155 & 158 & 143 & 135 \\
\hline Average & 347 & 253 & 236 & 247 & 212 & 184 \\
\hline
\end{tabular}

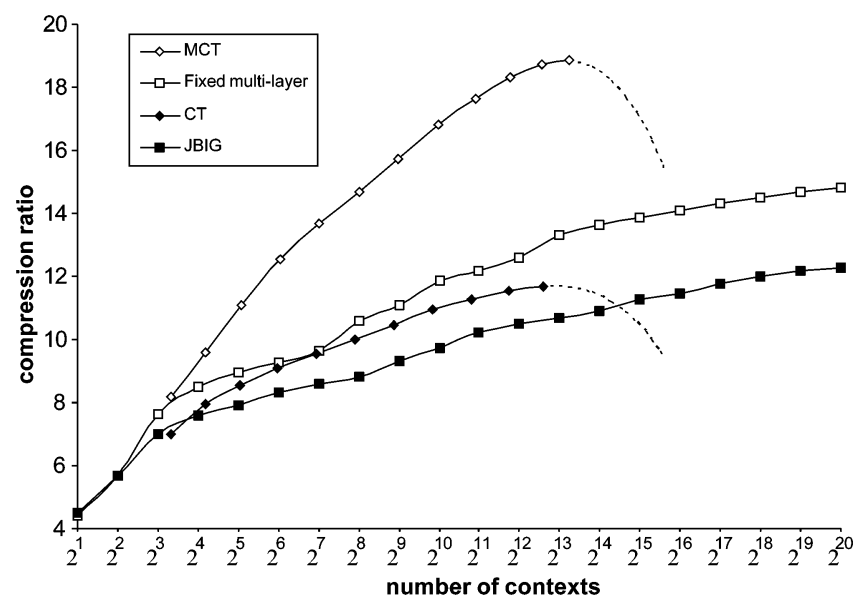

Fig. 17. Compression performance as a function of the number of contexts. The results are for the basic layer of the image 431204 (with semantic separation). In the case of multilayer compression, water is used as a reference layer.

and fixed multilayer context (Fixed multilayer). In this experiment, the size of the context tree was explicitly forced to given size (solid line) and also to grow beyond the stopping criterion (the dashed line) for comparison purposes. The results indicate that better compression can be obtained by increasing the context size (JBIG curve), by using context tree modeling (CT versus JBIG), and by using multilayer contexts (Fixed multilayer versus JBIG). Nevertheless, the joint application of them 
TABLE VI

COMPRESSION AND DECOMPRESSION TIMES USING 500 MHz PENTIUM III

\begin{tabular}{|c|c|c|c|c|c|c|c|c|}
\hline & \multicolumn{2}{|c|}{ JBIG } & \multicolumn{2}{|c|}{ CT } & \multicolumn{4}{|c|}{ MCT } \\
\hline & Comp. & Decomp. & $\begin{array}{l}\text { Tree + } \\
\text { Comp. }\end{array}$ & Decomp & $\begin{array}{c}\text { Cost } \\
\text { matrix }\end{array}$ & $\begin{array}{l}\text { Optimal } \\
\text { ordering }\end{array}$ & Comp. & Decomp \\
\hline emantic & $0: 02: 23$ & $0: 02: 09$ & $0: 45: 02$ & $0: 05: 38$ & $61: 34: 00$ & $0: 00: 04$ & $0: 03: 10$ & $0: 05: 39$ \\
\hline Set \#1 & $0: 03: 44$ & & $1: 39: 27$ & & $111: 59: 00$ & & $0: 06: 23$ & $0: 11: 05$ \\
\hline Set \#2 & $0: 00: 09$ & $0: 00: 10$ & $0: 02: 35$ & $0: 00: 18$ & $2: 46: 00$ & $0: 00: 04$ & $0: 00: 10$ & $0: 00: 18$ \\
\hline Set \#3 & $0: 00: 22$ & $0: 00: 23$ & $0: 08: 58$ & 0:01:03 & $28: 16: 00$ & & $0: 00: 45$ & 0:01:03 \\
\hline Set \#4 & $0: 00: 22$ & $0: 00: 24$ & $0: 09: 23$ & 0:01:07 & $33: 02: 00$ & $0: 00: 04$ & $0: 00: 38$ & $0: 01: 07$ \\
\hline Set \#5 & $0: 00: 08$ & & $0: 02: 51$ & $0: 00: 21$ & $22: 48: 00$ & $0: 00: 02$ & $0: 00: 12$ & $0: 00: 21$ \\
\hline Sum & $0: 07: 06$ & $0: 06: 50$ & $2: 45: 28$ & $0: 19: 29$ & $260: 25: 00$ & $0: 00: 23$ & $0: 11: 18$ & $0: 19: 31$ \\
\hline
\end{tabular}

with the optimized layer ordering gives significantly better compression than any of the ideas alone.

The improved compression, however, does not come without a price. In this case, the drawback is a significantly increased compression time (see Table VI). Most of the time is spent for calculating the cost matrix, i.e., tentatively compressing the image layers using all possible current-reference layer pairs. Finding the optimal ordering does not take much time at all, although the algorithm itself has the time complexity of $O\left(n^{4}\right)$. The actual compression is somewhat slower than that of the CT but the difference is tolerable. In comparison to the JBIG, both the $\mathrm{CT}$ and $\mathrm{MCT}$ are somewhat slower also in the decompression.

For illustrating the performance of the CT and MCT in detail, we have collected four most used contexts and their statistics in Fig. 18. In the case of independent compression, the optimized context trees can be remarkably different for different layers. For example, the contexts in fields layer contain much fewer pixels than the contexts in contours layer. Pixels are also taken further away and only a few nearby pixels are needed to model the probability distribution. The contours layer does not contain large convex shapes as the fields layers, but more complex thin lines that need more context pixels to be modeled accurately.

In the case of multilayer context tree, the selection of the context pixels depends on the layers in question. The general tendency is the same as in the case of independent compression so that fields and water layers need fewer context pixels. The basic layer uses significantly more pixels although the number of pixels depends on the particular pixel combination. The number of the context pixels in the reference layer is typically much smaller. When the basic layer is used as reference, only a few nearby pixels are used. In the case of water, on the other hand, the context pixels are scattered from wider area.

Finally, the result of the proposed method (MCT) is compared with the following methods:

1) GIF: CompuServe graphics interchange format [1];

2) PNG: portable network graphics [2], [3];

3) JBIG: joint bi-level image group [6];

4) PPM: prediction by partial matching [11], [12];

5) PWC: piecewise-constant image model [13];

6) SKIP: explicit skipping of pixels set in previous layer [10];

7) CT: single layer context tree [16], [22].

The results are summarized in Table VII (semantic layers) and Table VIII (color separation). Note that the results of GIF, PNG, PPM, and PWC are applied to the color images and the JBIG, SKIP, CT, and MCT for the binary layers. SKIP has been designed for color separation where the exclusion of certain pixels

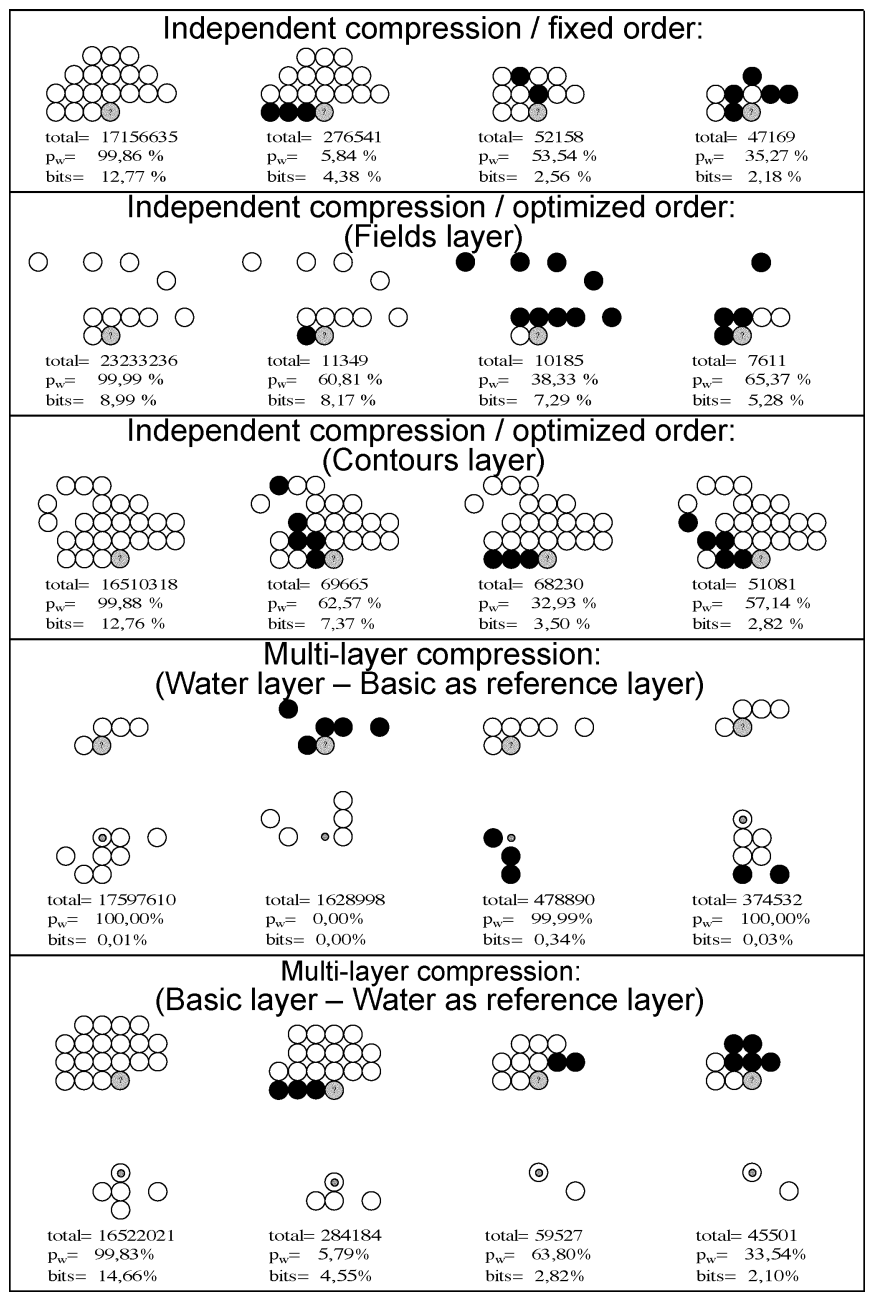

Fig. 18. Most used contexts in various context tree modeling strategies when applied to the Set \#1 with semantic separation.

TABLE VII

COMPRESSION RESULTS (KILOBYTES) FOR THE METHODS IN COMPARISON FOR SEMANTIC SEPARATION

\begin{tabular}{|c|c|c|c|c|c|c|c|c|}
\hline $\begin{array}{l}\text { Semantic } \\
\text { separation }\end{array}$ & GIF & PNG & JBIG & PPM & PWC & SKIP & CT & MCT \\
\hline \begin{tabular}{|l|}
124101 \\
\end{tabular} & 610 & 865 & 175 & 429 & 238 & 183 & 156 & 132 \\
\hline 201401 & 2642 & 3953 & 791 & 2287 & 1238 & 933 & 685 & 599 \\
\hline 263112 & 1028 & 1416 & 277 & 797 & 424 & 337 & 246 & 223 \\
\hline 431204 & 2295 & 3158 & 650 & 1796 & 1009 & 799 & 569 & 451 \\
\hline 431306 & 2431 & 3275 & 656 & 1936 & 958 & 743 & 549 & 508 \\
\hline Average & 1801 & 2533 & 510 & 1449 & 774 & 599 & 441 & 382 \\
\hline $\begin{array}{c}\text { Bits per } \\
\text { pixel }\end{array}$ & 0,590 & 0,830 & 0,167 & 0,475 & 0,253 & 0,196 & 0,145 & 0,125 \\
\hline
\end{tabular}

TABLE VIII

COMPRESSION RESULTS (KILOBYTES) FOR THE METHODS IN COMPARISON FOR COLOR SEPARATION

\begin{tabular}{|c|c|c|c|c|c|c|c|c|}
\hline $\begin{array}{c}\text { Color } \\
\text { separation }\end{array}$ & GIF & PNG & JBIG & PPM & PWC & SKIP & СT & МСТ \\
\hline Set\#1 & 1801 & 2533 & 1017 & 1449 & 774 & 532 & 616 & 401 \\
\hline Set \#2 & 86 & 90 & 62 & 81 & 35 & 34 & 34 & 30 \\
\hline Set \#3 & 288 & 278 & 283 & 203 & 198 & 202 & 185 & 155 \\
\hline Set \#4 & 303 & 287 & 274 & 211 & 197 & 198 & 240 & 198 \\
\hline Set \#5 & 188 & 184 & 210 & 143 & 139 & 145 & 158 & 135 \\
\hline Average & 533 & 674 & 369 & 417 & 269 & 222 & 247 & 184 \\
\hline $\begin{array}{l}\text { Bits per } \\
\text { pixel }\end{array}$ & 1,655 & 1,659 & 1,571 & 1,234 & 1,079 & 1,085 & 1,162 & 0,970 \\
\hline
\end{tabular}

can be concluded from the existence of the pixel in previous layer. In semantic separation, the SKIP can also be applied if we assume the order of the layers to be presented on the final color 
image. Effectively, we are then doing color separation with a predefined order of compressing the layers.

The result shows that the MCT gives the best compression with all test sets. In the case of color separation, the SKIP method is rather effective in comparison to JBIG despite of its simplicity. Also, PWC and CT perform rather well. In the case of semantic separation, the binary image coding methods (JBIG, SKIP, CT, and MCT) are clearly better than the others, of which the MCT gives most compression.

\section{CONCLUSION}

We have proposed a method for compressing map images by multilayer context tree modeling and by optimizing the order of the processing of the binary layers. Solutions are given for the context modeling, utilization of the multilayer dependencies and for the optimal ordering of the layers. The proposed method gives about $25 \%$ improvement over previous methods in comparison to the compression of binary layers without utilizing the interlayer dependencies. The optimal order of processing the layers was considered as directed spanning tree problem and solved by an algorithm derived from the Edmond's algorithm.

The proposed method requires a lot of processing in the encoding. Therefore, it is not suitable for on-line applications where the encoding must be done real time. The decoding stage, however, does not require any additional processing. The proposed method is therefore suitable for applications where encoding is done only once and without any such limitations for the decoding part.

There are ideas not dealt in this paper that could be considered in the future. For example, the two-layer context modeling could be generalized to 3-D by conditioning the probability of the pixel on several layers instead of only one. However, it is not obvious how the optimal order of layers should then be solved, and whether this modification would result additional compression worth the trouble. Another idea would be to model the image as a color image instead of dividing it into binary layers. In this case, the context modeling would become significantly more complex. A third idea worth further studies might be to develop the method towards real-time compression by designing faster heuristic for estimating the interlayer dependencies instead of the brute force calculation of the cost matrix.

\section{REFERENCES}

[1] T. A. Welch, "A technique for high-performance data compression," Computer, vol. 17, no. 6, pp. 8-19, June 1984.

[2] P. Deutsch. (May 1996) DEFLATE compressed data format specification. [Online]http://www.cis.ohio-state.edu/htbin/rfc/rfc1951.html

[3] J. Ziv and A. Lempel, "A universal algorithm for sequential data compression," IEEE Trans. Inf. Theory, vol. 23, no. 3, pp. 337-343, May 1977.

[4] W. B. Pennebaker and J. L. Mitchell, JPEG Still Image Data Compression Standard. New York: Van Nostrand Reinhold, 1993.

[5] M. Weinberger, G. Seroussi, G. Sapiro, and M. W. Marcellin, "The LOCO-I lossless image compression algorithm: Principles and standardization into JPEG-LS," HP Laboratories, HPL98-193, 1998.

[6] JBIG: ISO/IEC Int. Std. 11544, 1993.
[7] P. G. Howard, F. Kossentini, B. Martins, S. Forchammer, and W. J. Rucklidge, "The emerging JBIG2 standard," IEEE Trans. Circuits Syst. Video Technol., vol. 8, no. 7, pp. 838-848, July 1998.

[8] G. G. Langdon and J. Rissanen, "Compression of black-white images with arithmetic coding," IEEE Trans. Commun., vol. 29, no. 6, pp. 858-867, June 1981.

[9] Y. Yoo, Y. Kwon, and A. Ortega, "Embedded image-domain adaptive compression of simple images," in Proc. 32nd Asilomar Conf. Signals, Systems, and Computers, Nov. 1998.

[10] S. Forchhammer and O. R. Jensen, "Content layer progressive coding of digital maps," IEEE Trans. Image Process., vol. 11, no. 12, pp. 1349-1356, Dec. 2002.

[11] J. G. Cleary and I. H. Witten, "Data compression using adaptive coding and partial string matching," IEEE Trans. Commun., vol. 32, no. 4, pp. 396-402, Apr. 1984.

[12] S. Forchhammer and J. M. Salinas, "Progressive coding of palette images and digital maps," in Proc. IEEE Data Compression Conf., Snowbird, UT, Apr. 2002, pp. 362-371.

[13] P. J. Ausbeck, Jr., "The piecewise-constant image model," Proc. IEEE, vol. 88, no. 11, pp. 1779-1789, Nov. 2000.

[14] E. I. Ageenko, P. Kopylov, and P. Fränti, "Optimizing context template for compression of multicomponent map images," in Proc. GraphiCon, Moscow, Russia, 2000, pp. 151-156.

[15] — - "On the size and shape of multilevel context templates for compression of map images," in IEEE Int. Conf. Image Processing, vol. 3, Thessaloniki, Greece, Oct. 2001, pp. 458-461.

[16] B. Martins and S. Forchhammer, "Bi-level image compression with tree coding," IEEE Trans. Image Process., vol. 7, no. 4, pp. 517-528, Apr. 1998.

[17] P. Kopylov and P. Fränti, "Context tree compression of multicomponent map images," in Proc. IEEE Data Compression Conf., Snowbird, UT, Apr. 2002, pp. 212-221.

[18] - "Optimal layer ordering in the compression of map images," in Proc. IEEE Data Compression Conf., Snowbird, UT, Apr. 2003, pp. 323-332.

[19] J. J. Rissanen and G. G. Langdon, "Universal modeling and coding," IEEE Trans. Inf. Theory, vol. IT-27, pp. 12-23, Jan. 1981.

[20] - "Arithmetic coding," IBM J. Res. Develop., vol. 23, pp. 146-162, 1979.

[21] C. E. Shanon, "A mathematical theory of communication," Bell Syst. Tech. J., vol. 27, pp. 398-403, 1948.

[22] P. Fränti and E. I. Ageenko, "On the use of context tree for binary image compression," in Proc. IEEE Int. Conf. Image Processing, vol. 3, Kobe, Japan, 1999, pp. 752-756.

[23] H. Helfgott and M. Cohn, "Linear-time construction of optimal context trees," in Proc. IEEE Data Compression Conf., Snowbird, UT, Apr. 1998, pp. 369-377.

[24] R. I. Nohre, "Topics in descriptive complexity," Ph.D. dissertation, Univ. Lingköping, Lingköping, Sweden, 1994.

[25] ESRI. (1998) ESRI shapefile technical description. An ESRI White Paper. [Online]. Available: http://www.esri.com/library/ whitepages/pdfs/shapefile.pdf

[26] National Land Survey of Finland, Helsinki, Finland. [Online]. Available: http://www.nls.fi/index_e.html

[27] E. I. Ageenko and P. Fränti, "Compression of large binary images in digital spatial libraries," Comput. Graph., vol. 24, no. 1, pp. 91-98, Feb. 2000.

[28] M. J. Weinberger, J. Rissanen, and R. Arps, "Application of universal context modeling to lossless compression of gray-scale images," IEEE Trans. Image Process., vol. 5, no. 4, pp. 575-586, Apr. 1996.

[29] M. Rabbani and P. W. Melnychuck, "Conditioning contexts for the arithmetic coding of bit planes," IEEE Trans. Signal Process., vol. 40, no. 11, pp. 232-236, Nov. 1992.

[30] S. R. Tate, "Band ordering in lossless compression of multispectral images," IEEE Trans. Comput., vol. 46, no. 4, pp. 477-483, Apr. 1997.

[31] T. Cormen, C. Leiserson, and R. Rivest, Introduction to Algorithms. Cambridge, MA: MIT, 1990.

[32] R. C. Prim, "Shortest connection networks and some generalizations," Bell Syst. Tech. J., vol. 36, pp. 1389-1401, 1957.

[33] J. Edmonds, "Optimum branchings," J. Res. Nat. Bur. Stand., vol. 71B, pp. 133-240, 1967.

[34] R. E. Tarjan, "Finding optimum branchings," Networks, vol. 7, pp. 25-35, 1977. 


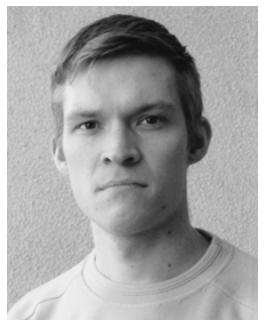

Pavel Kopylov receiced the M.Sc. degree from Moscow State University, Ulyanovsk, Russia, in 1997 and the Ph.D. degree from the University of Joensuu, Finland, in 2004.

Since 2000, he has been a Postdoctoral student at the University of Joensuu, Joensuu, Finland. His primary research interests are in image compression and restoration techniques.

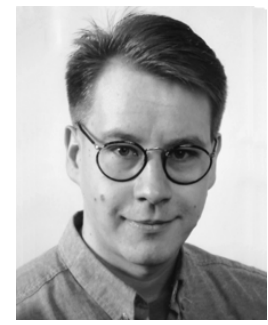

Pasi Fränti received the M.Sc. and Ph.D. degrees in computer science from the University of Turku, Finland, in 1991 and 1994, respectively.

From 1996 to 1999, he was a Postdoctoral Researcher with the University of Joensuu, Joensuu, Finland (funded by the Academy of Finland), where he has been a Professor since 2000. His primary research interests are in image compression, vector quantization, and clustering algorithms. 